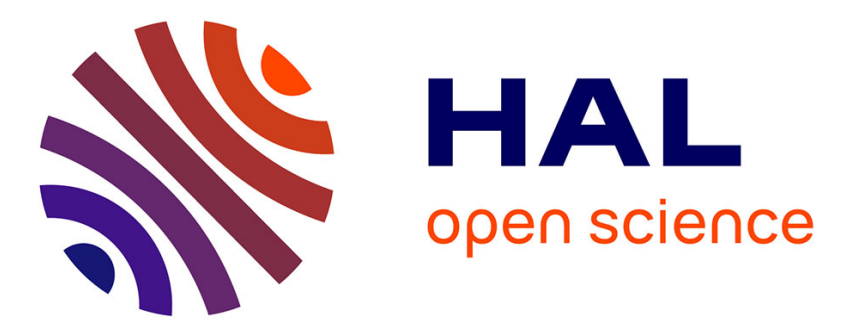

\title{
Epitaxie en phase vapeur par pyrolyse d'organométalliques (EPVOM) des solutions solides ternaires Ga1-xAl xSb, Ga1-xInxSb et GaAsySb1-y sur substrats de GaSb
}

G. Bougnot, J. Bougnot, F. Delannoy, A. Foucaran, P. Grosse, M. Marjan, F. Pascal, F. Roumanille

\section{To cite this version:}

G. Bougnot, J. Bougnot, F. Delannoy, A. Foucaran, P. Grosse, et al.. Epitaxie en phase vapeur par pyrolyse d'organométalliques (EPVOM) des solutions solides ternaires Ga1-xAl xSb, Ga1-xInxSb et GaAsySb1-y sur substrats de GaSb. Revue de Physique Appliquée, 1987, 22 (8), pp.837-844. 10.1051/rphysap:01987002208083700 . jpa-00245617

\section{HAL Id: jpa-00245617 https://hal.science/jpa-00245617}

Submitted on 1 Jan 1987

HAL is a multi-disciplinary open access archive for the deposit and dissemination of scientific research documents, whether they are published or not. The documents may come from teaching and research institutions in France or abroad, or from public or private research centers.
L'archive ouverte pluridisciplinaire HAL, est destinée au dépôt et à la diffusion de documents scientifiques de niveau recherche, publiés ou non, émanant des établissements d'enseignement et de recherche français ou étrangers, des laboratoires publics ou privés. 


\title{
Epitaxie en phase vapeur par pyrolyse d'organométalliques (EPVOM) des solutions solides ternaires $\mathbf{G a}_{1-x} \mathbf{A l}_{x} \mathbf{S b}, \mathbf{G a}_{1-x} \mathbf{I n}_{x} \mathbf{S b}$ et $\mathrm{GaAs}_{\boldsymbol{y}} \mathrm{Sb}_{1-\boldsymbol{y}}$ sur substrats de GaSb (*)
}

\author{
G. Bougnot, J. Bougnot, F. Delannoy, A. Foucaran, P. Grosse, M. Marjan, F. Pascal et \\ F. Roumanille
}

Centre d'Electronique de Montpellier (UA CNRS 391), Université des Sciences et Techniques du Languedoc, pl. E.-Bataillon, 34060 Montpellier Cedex, France

(Reçu le 16 janvier 1987, accepté le 4 mars 1987)

\begin{abstract}
Résumé. - Les solutions solides ternaires $\mathrm{Ga}_{1-x} \mathrm{Al}_{x} \mathrm{Sb}, \mathrm{Ga}_{1_{-}} \mathrm{In}_{x} \mathrm{Sb}$ et $\mathrm{GaAs}_{y} \mathrm{Sb}_{1_{-}}$ont été élaborées par épitaxie en phase vapeur par pyrolyse d'organométalliques (EPVOM), sur substrats de GaSb. On décrit l'évolution de la morphologie des couches avec le désaccord de paramètre de maille par rapport au substrat. Dans $\mathrm{Ga}_{1-x} \mathrm{Al}_{x} \mathrm{Sb}$ et $\mathrm{Ga}_{1-x} \mathrm{In}_{x} \mathrm{Sb}(x \leqslant 0,50)$, la relation entre la composition $x$ du solide et la composition correspondante $x_{\mathrm{v}}$ de la phase vapeur pour différentes températures de croissance est étudiée expérimentalement. Une tentative d'explication de la variation importante de $x$ avec la température est effectuée en s'appuyant sur un modèle thermodynamique simple. Dans le cas de $\mathrm{GaAs}_{y} \mathrm{Sb}_{1-y}$, l'influence du rapport $\mathrm{As} / \mathrm{Sb}$ et du rapport $\mathrm{As}+\mathrm{Sb} / \mathrm{Ga}$ dans la phase vapeur sur la composition $y$ du solide est également présentée. Les résultats confirment la formation, par EPVOM, de la solution solide GaAsSb dans le domaine métastable.
\end{abstract}

\begin{abstract}
The $\mathrm{Ga}_{1_{-x}} \mathrm{Al}_{x} \mathrm{Sb}, \mathrm{Ga}_{1-x} \mathrm{In}_{x} \mathrm{Sb}$ and $\mathrm{GaAs}_{y} \mathrm{Sb}_{1_{-y}}$ ternary solid solutions have been successfully grown by metal organic vapour phase epitaxy (MOVPE) on GaSb substrates. Surface morphology of the epilayers is first described in terms of lattice mismatch with the substrate. For both $\mathrm{Ga}_{1-x} \mathrm{Al}_{x} \mathrm{Sb}$ and $\mathrm{Ga}_{1-x} \mathrm{In}_{x} \mathrm{Sb}(x \leqslant 0.5)$, the solid composition dependence on the corresponding vapor phase composition $x_{\mathrm{v}}$ is experimentally explored for different growth temperatures. The important variation of $x$ with temperature at given $x_{\mathrm{v}}$ is tentatively explained using a simple thermodynamical model. For $\mathrm{GaAs}_{y} \mathrm{Sb}_{1-y}$, the influence of $\mathrm{As} / \mathrm{Sb}$ and $\mathrm{V}$ elements/III partial pressure ratios on the solid composition is also investigated. Finally, it is confirmed that $\mathrm{GaAs}_{y} \mathrm{Sb}_{1-y}$ can be grown by MOVPE into the miscibility gap.
\end{abstract}

\section{Introduction.}

Les alliages quaternaires $\mathrm{Ga}_{1-x} \mathrm{Al}_{x} \mathrm{As}_{y} \mathrm{Sb}_{1-y}$ et $\mathrm{Ga}_{1-x} \mathrm{In}_{x} \mathrm{As}_{y} \mathrm{Sb}_{1-y}$ possèdent des bandes interdites comprises entre 2,16 et $0,73 \mathrm{eV}$ et 1,43 et $0,10 \mathrm{eV}$ respectivement, couvrant ainsi les longueurs d'onde intéressant les télécommunications par fibres optiques $-1,3 \mu \mathrm{m}-1,55 \mu \mathrm{m}$ et la gamme 2,5-4 $\mu \mathrm{m}$. Ils peuvent de plus s'épitaxier en accord de paramètre de maille sur GaSb, InAs et InP. Mais pour des compositions réalisant l'accord de maille sur InP ou sur InAs, InP et $\mathrm{GaSb}$ respectivement, ils présentent des zones de non-miscibilité $\left[\begin{array}{llll}1 & \text { à } & 4 & \text { pour }\end{array}\right.$ $\mathrm{Ga}_{1-x} \mathrm{Al}_{x} \mathrm{As}_{y} \mathrm{Sb}_{1-y}$ ] [5 à 8 pour $\mathrm{Ga}_{1-x} \mathrm{In}_{x} \mathrm{As}_{y} \mathrm{Sb}_{1-y}$ ]. Cependant en raison des fortes sursaturations qu'elles permettent de réaliser, des techniques

$\left(^{*}\right)$ Travail soutenu par la D.A.I.I. comme l'épitaxie par jets moléculaires (EJM) $[9,10]$ et l'épitaxie en phase vapeur par pyrolyse d'organométalliques (EPVOM) [11-14] ont effectivement donné des dépôts de $\mathrm{GaAs}_{y} \mathrm{Sb}_{1-y}$ sur substrats InP, $\mathrm{GaAs}$ ou $\mathrm{Al}_{2} \mathrm{O}_{3}$ dans le domaine métastable ; Jen et al. [15] signalent cependant, pour $y \simeq 0,50$ et sur substrat InP, la formation par EPVOM de structures ordonnées de type chalcopyrite ou tétragonal, structures qui seraient thermodynamiquement stables.

En ce qui concerne la solution solide $\mathrm{Ga}_{1-x} \mathrm{In}_{x} \mathrm{As}_{y} \mathrm{Sb}_{1-y}$, seuls Stringfellow et al. [16] ont réussi à déposer par EPVOM des couches ayant des compositions à l'intérieur du domaine de non-miscibilité, sur substrats GaSb et InAs alors que l'épitaxie en phase liquide est toujours limitée à $x \leqslant 0,23$ [1719]; enfin récemment des photodiodes

$$
\mathrm{Ga}_{0,82} \mathrm{In}_{0,18} \mathrm{As}_{0,17} \mathrm{Sb}_{0,83} / \mathrm{GaSb} \text {, }
$$


élaborées par épitaxie en phase liquide sur $\mathrm{GaSb}[20]$, présentent pour la première fois une réponse spectrale comprise entre 1,7 et $2,3 \mu \mathrm{m}$ avec un rendement quantique maximum de $43 \%$ à $2,2 \mu \mathrm{m}$ à polarisation nulle, à température ambiante.

On présente ici de nouveaux résultats portant sur la cristallogenèse des alliages ternaires $\mathrm{Ga}_{1-x} \mathrm{Al}_{x} \mathrm{Sb}$, $\mathrm{GaAs}_{y} \mathrm{Sb}_{1-y}$ et $\mathrm{Ga}_{1-x} \mathrm{In}_{x} \mathrm{Sb}$ déposés sur substrats de $\mathrm{GaSb}$ par pyrolyse d'organométalliques et d'arsine. Il s'agit, à notre connaissance, des premiers dépôts de GaAsSb et de GaInSb sur substrats de GaSb par EPVOM.

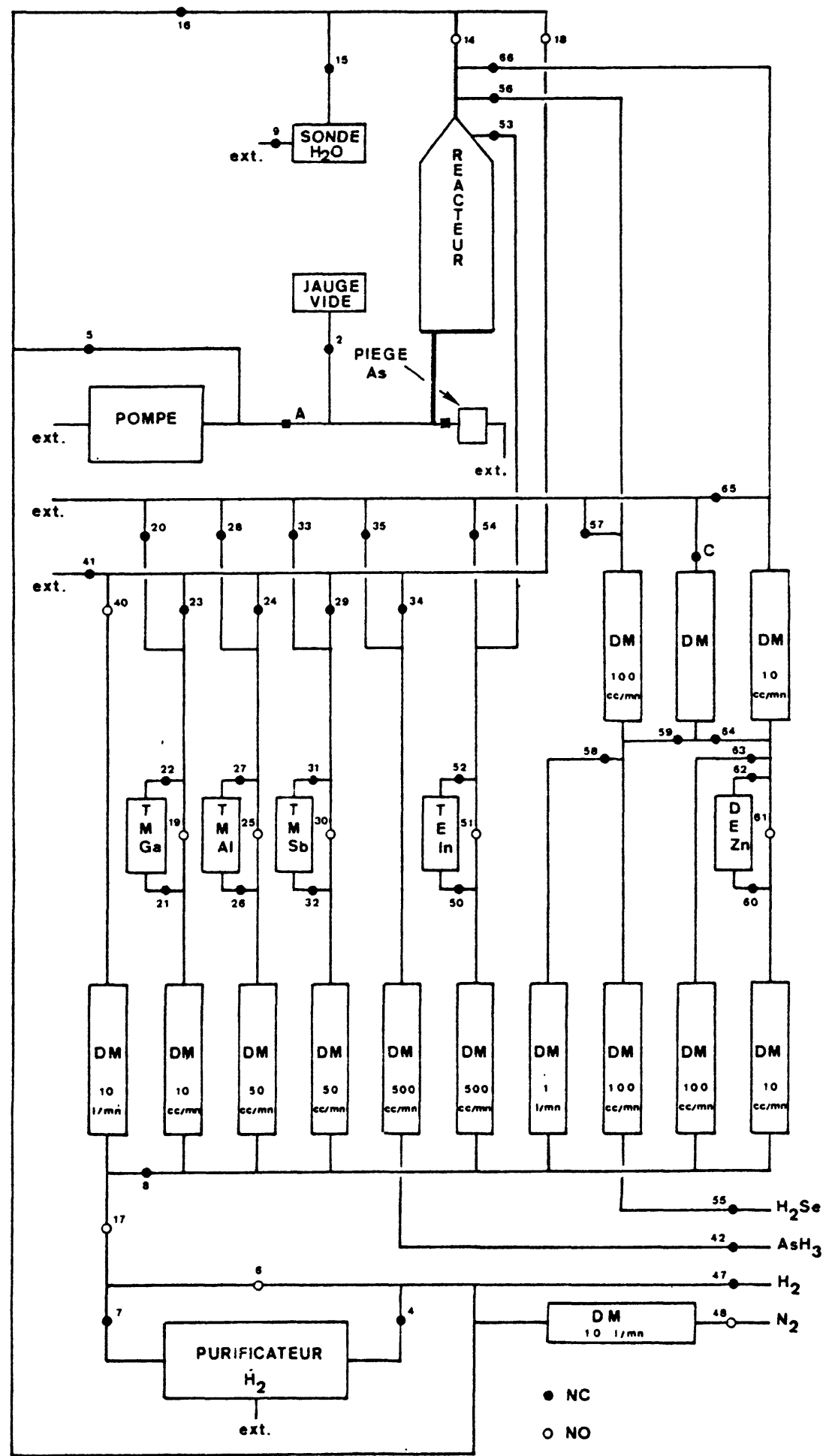

Fig. 1. - Schéma du panneau de gaz et du réacteur.

[Schematic diagram of the gas handling system and reactor.] 


\section{Conditions expérimentales.}

Le schéma du dispositif expérimental est représenté sur la figure 1 . Le réacteur est de type vertical à paroi refroidie, de diamètre intérieur $50 \mathrm{~mm}$. Le suscepteur en graphite, à face horizontale, est chauffé par H.F. ; les organométalliques utilisés sont le triméthyl gallium (TMG), le triméthyl aluminium (TMA), le triméthyl antimoine (TMSb) et le triéthyl indium (TEI); l'arsenic est apporté sous forme d'arsine (diluée à $1 \%$ dans $\mathrm{H}_{2}$ ). Les substrats de $\mathrm{GaSb}$ sont généralement d'orientation (100) et ils subissent avant introduction dans le réacteur un décapage au brome-méthanol.

Pendant la montée en température dans le réacteur, la pression de travail en TMSb est établie dès que la température dépasse $400^{\circ} \mathrm{C}$ afin d'éviter une évaporation d'antimoine.

Le tableau I rassemble les valeurs ou les gammes de valeurs choisies pour les paramètres expérimentaux ; notamment la somme des pressions partielles des organométalliques d'éléments III, $\sum P_{\mathrm{III}}$, (pour $\mathrm{GaAlSb}$ et GaInSb) et celle de $\mathrm{TMSb}$ et $\mathrm{AsH}_{3}$, $\sum P_{\mathrm{v}}$, (pour GaAsSb) est indiquée; le rapport $R=\sum P_{\mathrm{v}} / \sum P_{\mathrm{III}}$ et les fractions molaires $x_{\mathrm{v}}=$ $\boldsymbol{P}_{\mathrm{TMA}} / \boldsymbol{P}_{\mathrm{TMA}}+\boldsymbol{P}_{\mathrm{TMG}}$ ou $\boldsymbol{x}_{\mathrm{v}}=\boldsymbol{P}_{\mathrm{TEI}} / \boldsymbol{P}_{\mathrm{TEI}}+\boldsymbol{P}_{\mathrm{TMG}}$ et $y_{\mathrm{v}}=P_{\mathrm{AsH}_{3}} / P_{\mathrm{AsH}_{3}}+P_{\mathrm{TMSb}}$ sont aussi donnés.

Les vitesses de croissance sont généralement comprises entre 0,02 et $0,1 \mu \mathrm{m} / \mathrm{min}$ pour des pressions partielles d'éléments III de l'ordre $10^{-4}$ atm.

\section{Résultats expérimentaux et discussion.}

Les fractions molaires $x_{\mathrm{s}}$ de AlSb (dans GaAlSb) ou de InSb (dans GaInSb) et $y_{\mathrm{s}}$ de GaAs dans GaAsSb sont déterminées par diffraction de rayons $\mathrm{X}$ et à la microsonde électronique. La photoluminescence des couches de $\mathrm{Ga}_{1-x} \mathrm{Al}_{x} \mathrm{Sb}$ à $2 \mathrm{~K}$ a été également utilisée pour la mesure de la composition. On observe un assez bon accord entre les diverses déterminations.
3.1 MORPHOLOGIE. - La surface des couches offre un aspect brillant (photo 1) ; cependant, une observation microscopique montre la présence de quelques figures de croissance de forme pyramidale à base rectangulaire pour des couches présentant un faible désaccord de maille avec le substrat de GaSb. L'origine de ces figures est mal établie ; il semble cependant qu'elles prennent naissance en cours de croissance. Lorsque le désaccord de maille devient important, $\left(\Delta a / a>10^{-2}\right)$, la morphologie de surface se dégrade sensiblement comme le montrent les photographies $2(\mathrm{abc})$ et $3(\mathrm{abc})$. Cette dégradation

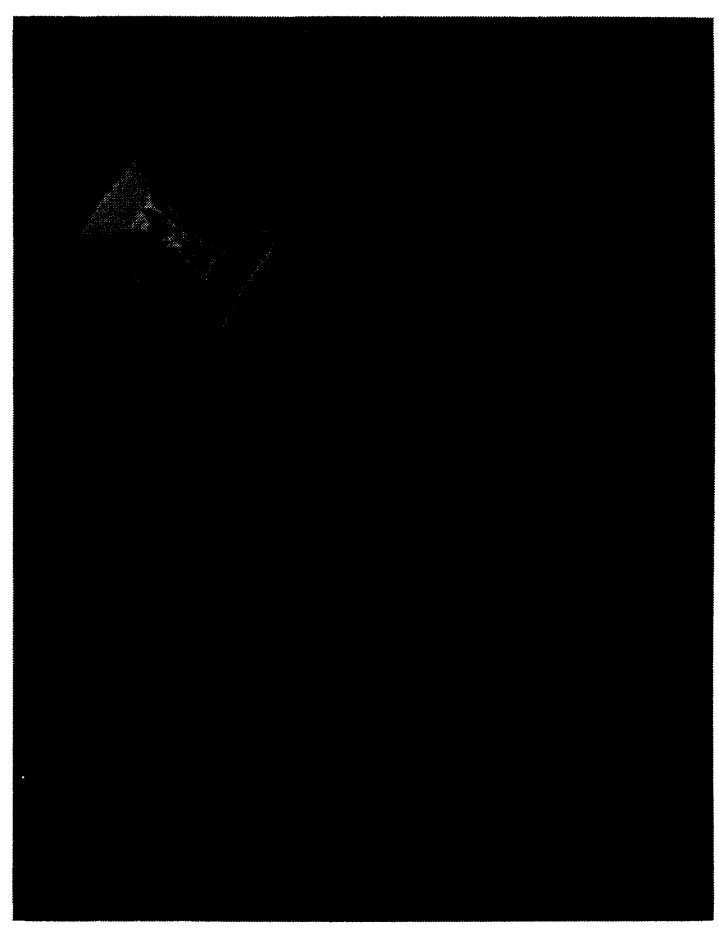

Photo 1. - Figure de croissance sur une couche de $\mathrm{Ga}_{0,89} \mathrm{Al}_{0,11} \mathrm{Sb}$ epitaxiée sur GaSb. Grossissement $G=500$.

[Hillock on a $\mathrm{Ga}_{0.89} \mathrm{Al}_{0.11} \mathrm{Sb}$ epilayer on GaSb. Magnification 500.]

Tableau I. - Paramètres de croissance.

[Growth parameters.]

\begin{tabular}{|c|c|c|c|}
\hline & $\mathrm{Ga}_{1-x} \mathrm{Al}_{x} \mathrm{Sb}$ & $\mathrm{Ga}_{1-x} \mathrm{In}_{x} \mathrm{Sb}$ & $\mathrm{GaAs}_{y} \mathrm{Sb}_{1-y}$ \\
\hline $\begin{array}{l}\text { Température de croissance }\left({ }^{\circ} \mathrm{C}\right) \\
\text { Débit } \mathrm{H}_{2} \text { entraîneur }(1 / \mathrm{min}) \\
\text { Température TMG }\left({ }^{\circ} \mathrm{C}\right) \\
\text { Température TMA }\left({ }^{\circ} \mathrm{C}\right) \\
\text { Température TMSb }\left({ }^{\circ} \mathrm{C}\right) \\
\text { Température TEI }\left({ }^{\circ} \mathrm{C}\right) \\
\sum P_{\mathrm{III}} \text { ou } \sum P_{\mathrm{v}}(\mathrm{atm}) \\
\text { Rapport V/III }=R \\
x_{\mathrm{v}} \text { ou } y_{\mathrm{v}}\end{array}$ & $\begin{array}{c}630-680 \\
1,25-3,5 \\
\left(0^{\circ} \mathrm{C}\right) \\
20 \\
-20 \\
0,5 \text { à } 2,5 \times 10^{-4} \\
1-8 \\
0 \text { à } 0,5\end{array}$ & $\begin{array}{c}500-700 \\
1,25-3,5 \\
\left(0^{\circ} \mathrm{C}\right) \\
-20 \\
30 \text { à } 60 \\
10^{4} \text { à } 4 \times 10^{-4} \\
0,2-2 \\
0 \text { à } 0,4\end{array}$ & $\begin{array}{c}550-680 \\
3,5 \\
\left(-8^{\circ} \mathrm{C}\right) \\
-20 \\
10^{-5} \text { à } 8 \times 10^{-4} \\
1-5 \\
0 \text { à } 0,4\end{array}$ \\
\hline
\end{tabular}




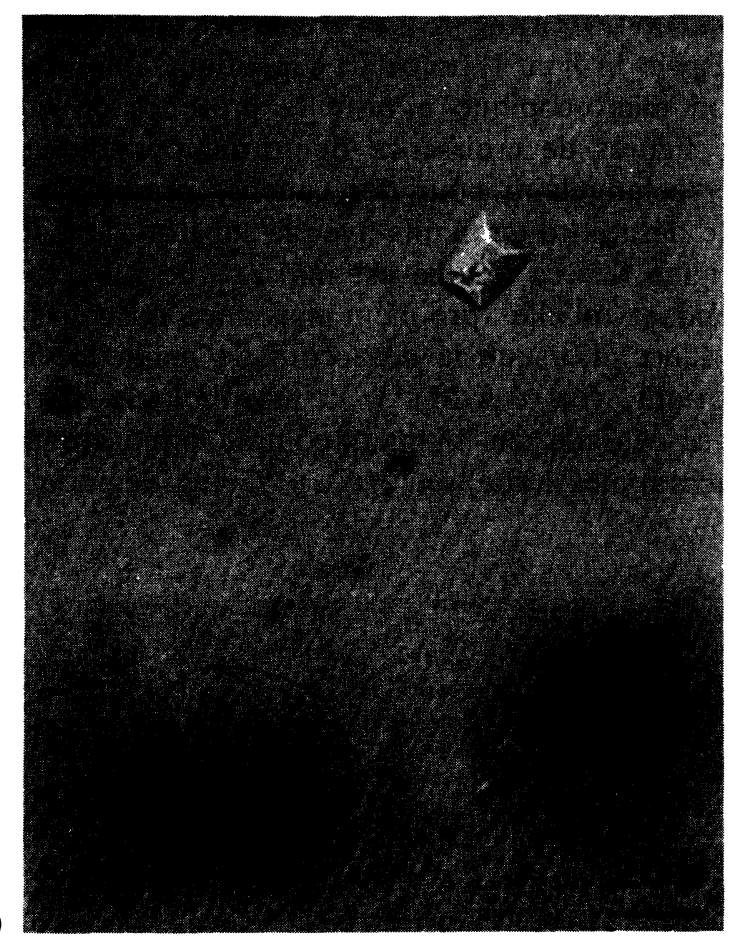

a)

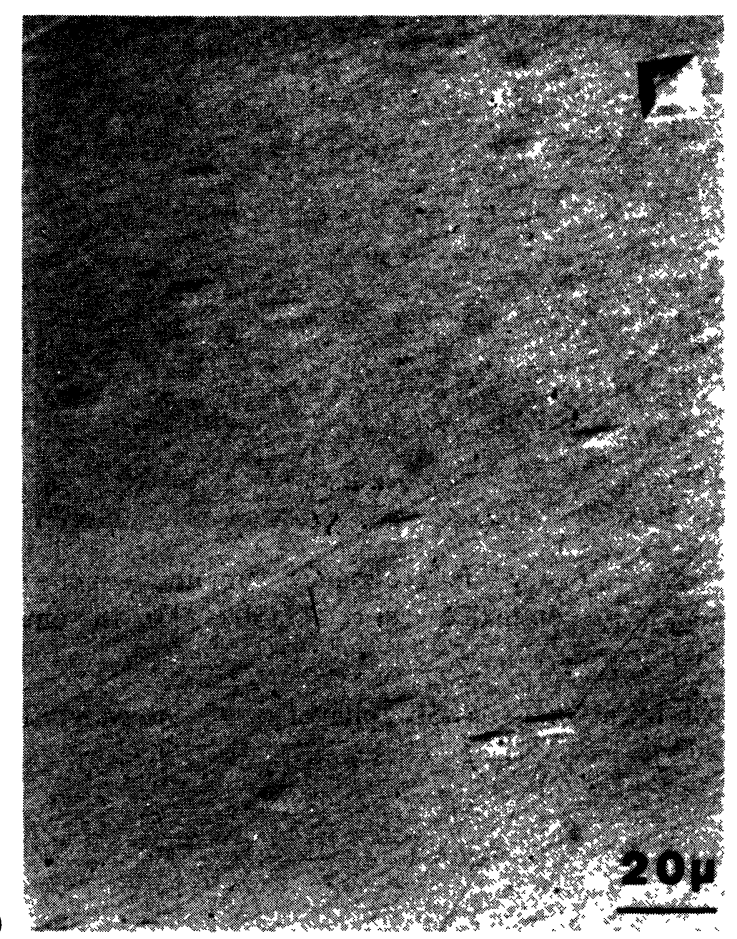

Photo 2. - Morphologie de surface de couches de $\mathrm{Ga}_{1-x} \mathrm{In}_{x} \mathrm{Sb}$ épitaxiées sur $\mathrm{GaSb}$. Grossissement 500 : (a) $x=0,18$, (b) $x=0,35$, (c) $x=0,58$.

[Surface morphology of $\mathrm{Ga}_{1-x} \operatorname{In}_{x} \mathrm{Sb}$ layers deposited on GaSb. Magnification 500 : (a) $x=0.18$, (b) $x=0.35$, (c) $x=0.58$.]

s'accompagne d'un élargissement très sensible des pics de rayons $X$; la figure 2 met en évidence ce phénomène sur $\mathrm{GaAs}_{y} \mathrm{Sb}_{1-y}$. Cependant, alors que dans $\mathrm{Ga}_{1-x} \mathrm{In}_{x} \mathrm{Sb}$, cette évolution doit être entière-

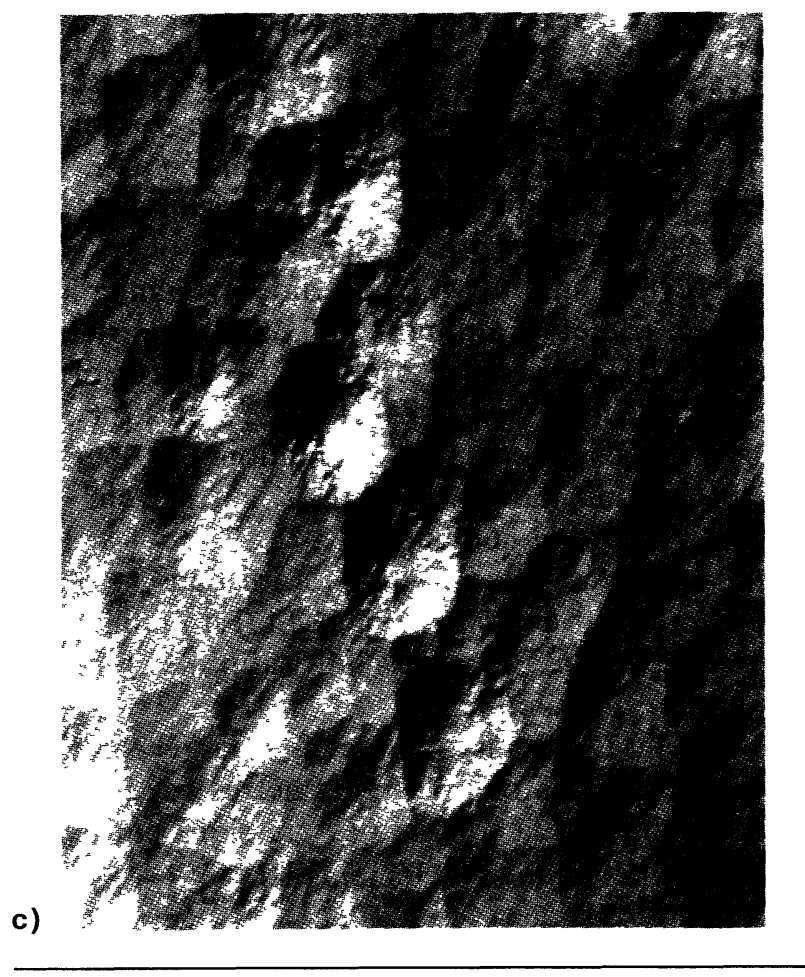

ment attribuée au désaccord de maille puisque la solution solide est stable dans tout le domaine de variation de $x$, dans $\mathrm{GaAs}_{y} \mathrm{Sb}_{1-y}$, l'existence de la zone de non-miscibilité intervient également ; il a en effet été observé dans ce cas des modulations de composition de très faible périodicité $[10,21]$ résultant vraisemblablement d'un début de décomposition spinodale.
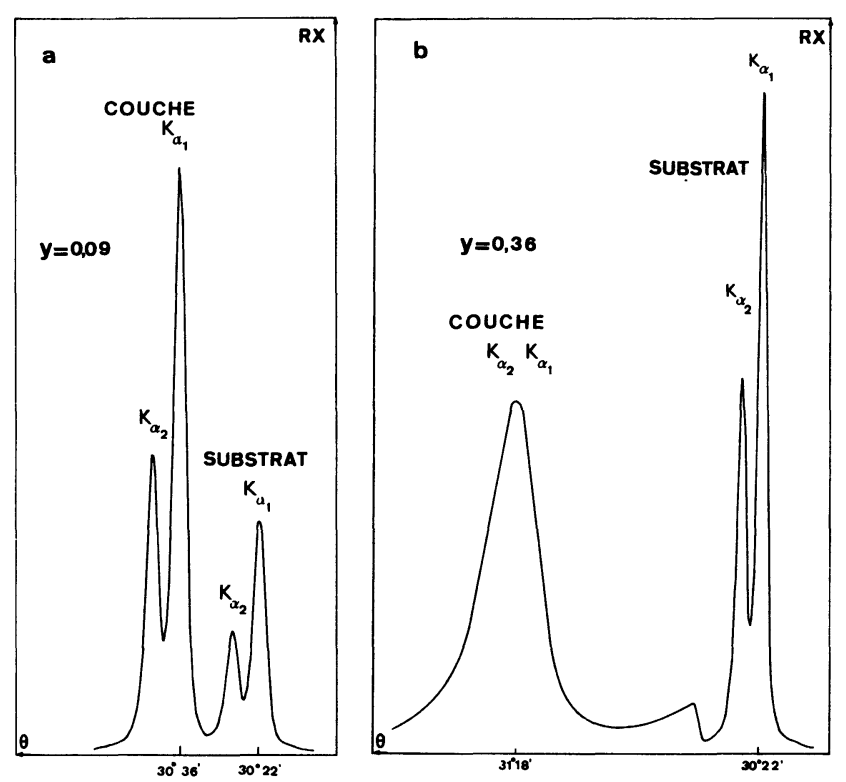

Fig. 2. - Spectres de diffraction de rayons $\mathrm{X}$ de couches de $\mathrm{GaAs}_{y} \mathrm{Sb}_{1-y}$ déposées sur GaSb : (a) dans la zone de miscibilité ; (b) hors de la zone de miscibilité.

[X-ray diffraction spectra of $\mathrm{GaAs}_{y} \mathrm{Sb}_{1-y}$ layers on $\mathrm{GaSb}$ : (a) outside the miscibility gap ; (b) inside the miscibility gap.] 
a)

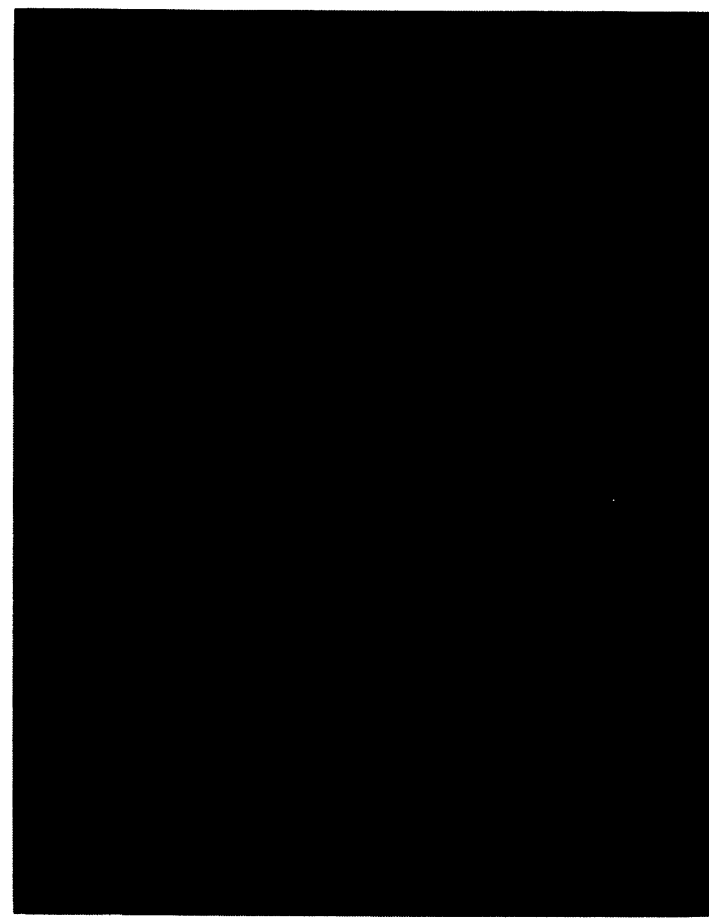

b)

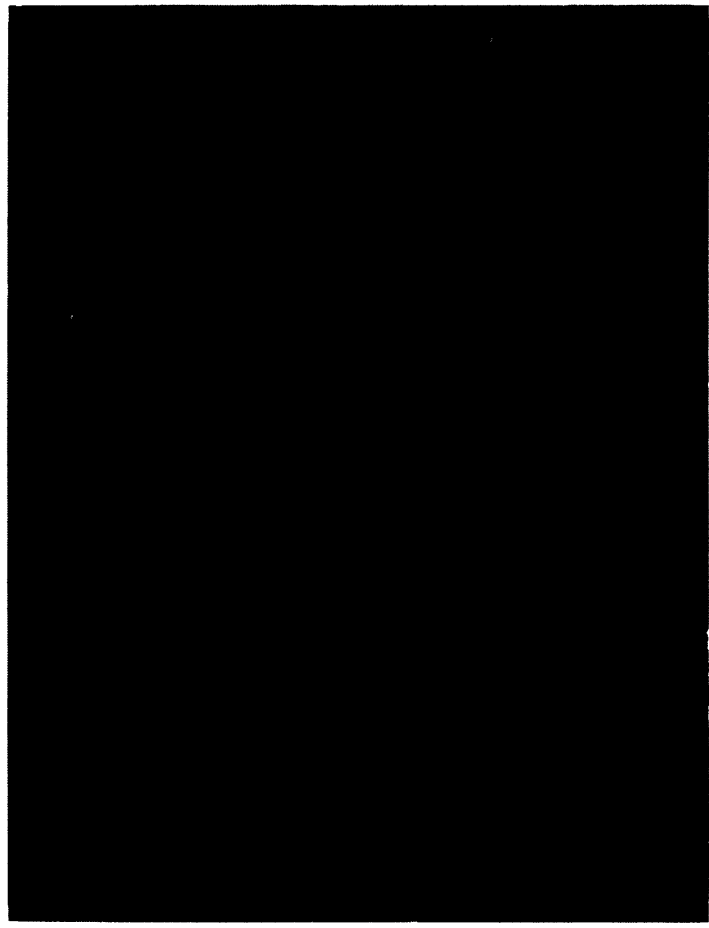

Photo 3. - Morphologie de surface de couches de $\mathrm{GaAs}_{y} \mathrm{Sb}_{1-y}$ épitaxiées sur $\mathrm{GaSb}$, grossissement $G=1000$ : (a) $y=0,09$, (b) $y=0,15$, (c) $y=0,31$.

[Surface morphology of $\mathrm{GaAs}_{y} \mathrm{Sb}_{1-y}$ layers deposited on GaSb, magnification : $1000:$ (a) $y=0.09$, (b) $y=0.15$, (c) $y=0.31$.]

\subsection{INFLUENCE DES PARAMÈTRES DE CROISSANCE} SUR LA COMPOSITION DES COUCHES. - $\mathrm{Ga}_{1-x} \mathrm{In}_{x} \mathrm{Sb}$ et $\mathrm{Ga}_{1-x} \mathrm{Al}_{x} \mathrm{Sb}$.

Les variations des fractions molaires $x_{\mathrm{s}}$ en fonction de la composition de la phase vapeur, $x_{\mathrm{v}}$, sont c)

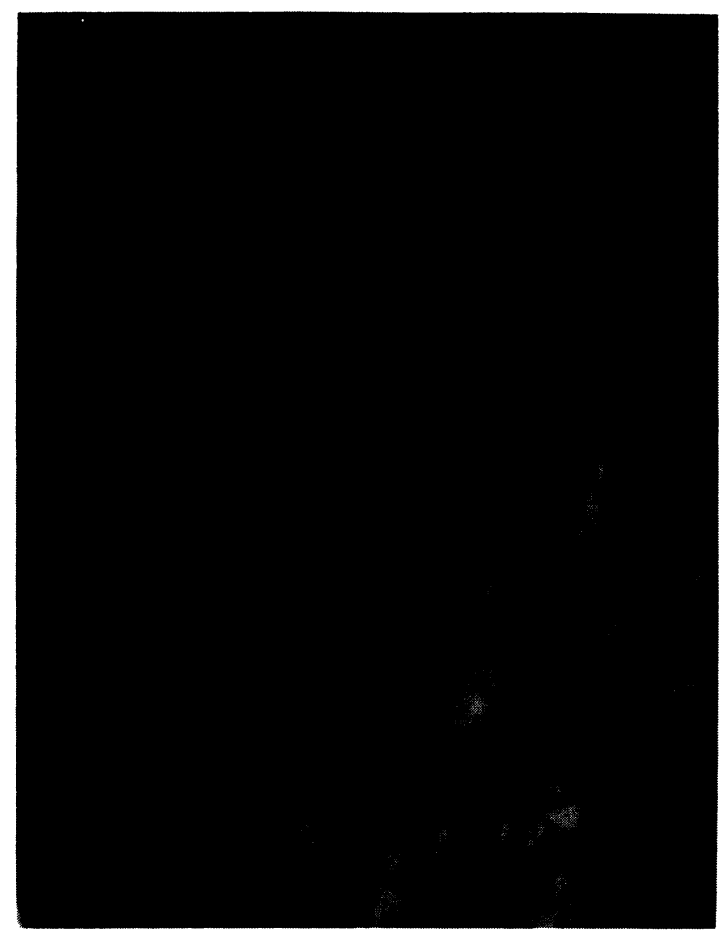

tracées sur les figures 3 et 4 pour $\mathrm{Ga}_{1-x} \mathrm{Al}_{x} \mathrm{Sb}$ et $\mathrm{Ga}_{1-x} \mathrm{In}_{x} \mathrm{Sb}$ respectivement.

On a également porté sur la figure 4, la courbe liquidus dans le plan pseudobinaire GaSb-InSb déduite de [22]; cette courbe représente, pour

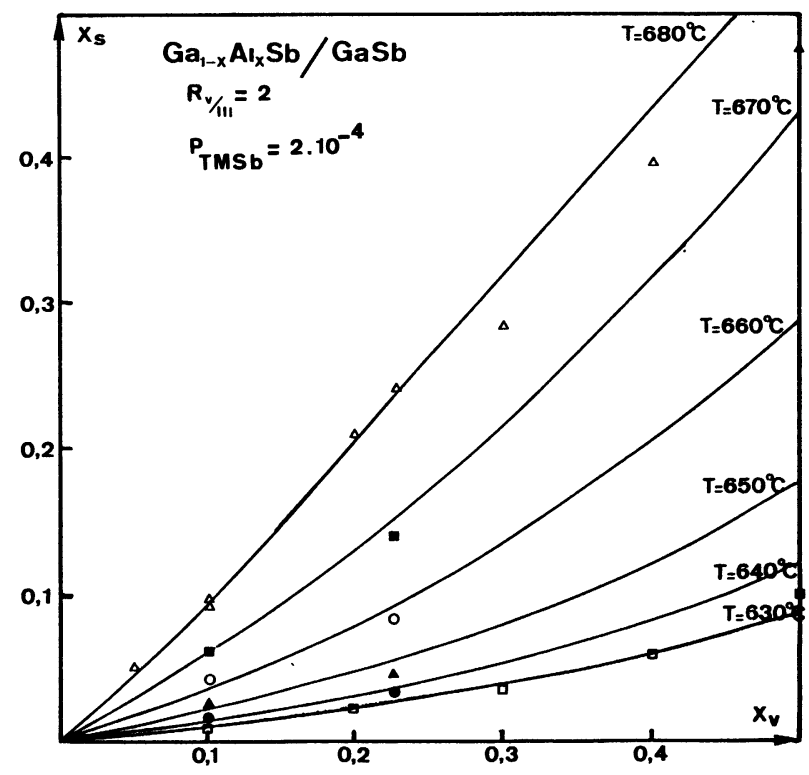

Fig. 3. - Fraction molaire $x_{\mathrm{s}}$ de AlSb dans le solide en fonction du rapport $x_{\mathrm{v}}=P_{\mathrm{TMA}} / P_{\mathrm{TMA}}+P_{\mathrm{TMG}}$ pour différentes températures d'épitaxie. Les courbes en traits pleins correspondent au modèle décrit dans le texte.

[AlSb molar fraction $x_{\mathrm{s}}$ in the solid as a function of the $x_{\mathrm{v}}=\boldsymbol{P}_{\mathrm{TMA}} / \boldsymbol{P}_{\mathrm{TMA}}+\boldsymbol{P}_{\mathrm{TMG}}$ ratio for different growth temperatures. The curves correspond to the model described in the text.] 


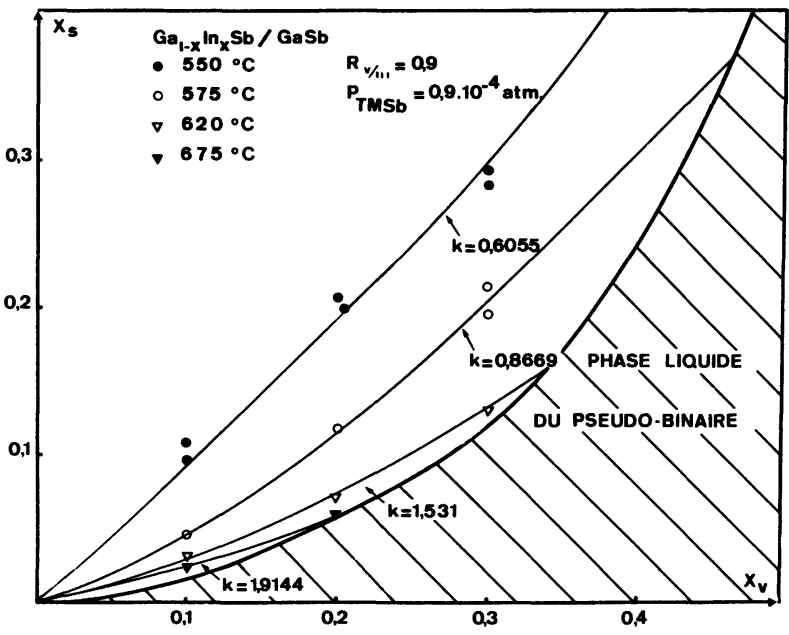

Fig. 4. - Fraction molaire $x_{\mathrm{s}}$ de InSb dans le solide en fonction du rapport $x_{\mathrm{v}}=P_{\mathrm{TEI}} / P_{\mathrm{TEI}}+P_{\mathrm{TMG}}$ pour différentes températures d'épitaxie. Les courbes en traits pleins correspondent au modèle décrit dans le texte.

[InSb molar fraction $x_{\mathrm{s}}$ in the solid as a function of the $x_{\mathrm{v}}=P_{\mathrm{TEI}} / P_{\mathrm{TEI}}+P_{\mathrm{TMG}}$ ratio for different growth temperatures. The curves correspond to the model described in the text.]

chaque valeur de la température, la composition $x$ au-delà de laquelle $\mathrm{Ga}_{1-x} \mathrm{In}_{x} \mathrm{Sb}$ ne peut être formé à l'état solide. On remarque qu'effectivement aucun dépôt homogène n'a pu être expérimentalement obtenu au-delà de cette limite.

Par ailleurs, on note que les rapports $x / x_{\mathrm{v}}$ sont inférieurs à 1 dans les gammes de température reportées - 630 à $680^{\circ} \mathrm{C}$ pour $\mathrm{GaAlSb}$ et 550 à $675^{\circ} \mathrm{C}$ pour $\mathrm{GaInSb}$. Ensuite, on constate que la fraction molaire $x$ de AlSb dans le solide diminue lorsque la température diminue alors qu'au contraire la fraction molaire de InSb croît. Ainsi les mesures mettent en évidence une influence importante de la température de croissance sur la composition $x$, à $x_{\mathrm{v}}$ donné.

Une première interprétation, essentiellement qualitative, et s'appuyant sur des considérations sur les cinétiques de croissance de GaSb, AlSb et InSb en fonction de la température, avait été précédemment avancée [23] ; on peut maintenant tenter de la préciser en utilisant un modèle thermodynamique simple, déjà proposé par Fukui et al. [24]. Dans le cas de $\mathrm{Ga}_{1-x} \mathrm{In}_{x} \mathrm{Sb}$ par exemple, ce modèle s'appuie sur les hypothèses suivantes :

* La pyrolyse des organométalliques et des hydrures est complète loin de l'interface,

* les équilibres thermodynamiques :

$$
\begin{aligned}
\mathrm{Ga}(\mathrm{g})+1 / 4 \mathrm{Sb}_{4}(\mathrm{~g}) & \rightleftharpoons \mathrm{GaSb}(\mathrm{s}) \\
\mathrm{In}(\mathrm{g})+1 / 4 \mathrm{Sb}_{4}(\mathrm{~g}) & \rightleftharpoons \mathrm{InSb}(\mathrm{s})
\end{aligned}
$$

sont réalisés à l'interface de croissance, les espèces gazeuses intervenant dans ces équilibres résultant de la pyrolyse des organométalliques. Les lois d'action de masse de ces deux équilibres s'écrivent :

$$
K_{\mathrm{GaSb}}=\frac{a_{\mathrm{GaSb}}}{P_{\mathrm{Ga}}\left(P_{\mathrm{Sb}_{4}}\right)^{1 / 4}}
$$

et

$$
K_{\mathrm{InSb}}=\frac{a_{\mathrm{InSb}}}{P_{\mathrm{In}}\left(P_{\mathrm{Sb}_{4}}\right)^{1 / 4}}
$$

où les $a_{i}$ et les $P_{i}$ représentent respectivement les activités et les pressions partielles d'équilibre à l'interface.

On néglige ainsi en première approximation les réactions en phase gazeuse susceptibles de former d'autres espèces comme $\mathrm{Sb}_{2}$ ou $\mathrm{SbH}_{3}$ par exemple. En effet, les calculs effectués sur d'autres systèmes montrent que c'est l'espèce tétra-atomique de l'élément $\mathrm{V}$ qui prédomine.

* Les proportions des éléments III se conservent dans la phase vapeur, ce qui se traduit par:

$$
\frac{P_{\mathrm{In}}^{0}}{P_{\mathrm{Ga}}^{0}}=\frac{P_{\mathrm{In}}}{P_{\mathrm{Ga}}}=\frac{x_{\mathrm{v}}}{1-x_{\mathrm{v}}}
$$

où les $P_{i}^{0}$ représentent les pressions partielles loin de l'interface.

Cette hypothèse consiste en fait à admettre que la diffusion des espèces' gazeuses In et $\mathrm{Ga}$ dans la couche limite voisine de l'interface s'effectue à la même vitesse.

* La solution solide $\mathrm{Ga}_{1-x} \mathrm{In}_{x} \mathrm{Sb}$ est considérée comme régulière ; les activités de $\mathrm{GaSb}$ et de $\mathrm{InSb}$ s'expriment alors en fonction du paramètre d'interaction $\omega_{\text {Gasb-Insb }}$ par les relations :

$$
\begin{aligned}
& a_{\text {InSb }}=x \exp \left[\omega_{\text {GaSb-InSb }}(1-x)^{2} / R T\right] \\
& a_{\text {GaSb }}=(1-x) \exp \left[\omega_{\text {GaSb-InSb }} x^{2} / R T\right] .
\end{aligned}
$$

Alors les équations (1) à (5) permettent d'écrire :

$$
\begin{aligned}
\frac{x}{1-x}= & \frac{x_{\mathrm{v}}}{1-x_{\mathrm{v}}} \frac{K_{\text {InSb }}}{K_{\mathrm{GaSb}}} \times \\
& \quad \times \exp \left[-(1-2 x) \omega_{\mathrm{GaSb}-\text { InSb }} / R T\right] .
\end{aligned}
$$

La même expression est obtenue pour $\mathrm{Ga}_{1-x} \mathrm{Al}_{x} \mathrm{Sb}$ avec $K_{\text {AISb }}$ remplaçant $K_{\text {InSb }}$ et $\omega_{\text {GaSb-AISb }}$ remplaçant $\omega_{\text {GaSb-InSb }}$; les valeurs de $\omega_{\text {Gasb-Insb }}$ et de $\omega_{\text {Gasb-Alsb }}$ sont celles calculées par Stringfellow à partir du modèle DLP (Delta Lattice Parameter) [25], soit respectivement 1846 et $23 \mathrm{cal}^{\mathrm{mole}}{ }^{-1}$.

Compte tenu de la grande incertitude sur les valeurs de $K_{\mathrm{GaSb}}, K_{\mathrm{InSb}}$ et $K_{\mathrm{AlSb}}$, les rapports $K=\frac{K_{\mathrm{GaSb}}}{K_{\mathrm{InSb}}}$ et $K=\frac{K_{\mathrm{GaSb}}}{K_{\mathrm{AISb}}}$ sont considérés comme des paramètres ajustables; en effet, des valeurs de $K_{\text {Insb }}$ aussi différentes que $244\left(\right.$ à $\left.500^{\circ} \mathrm{C}\right)$ [26] et $\left.1,2 \times 10^{15}(a ̀) 475^{\circ} \mathrm{C}\right)$ [27] ont été avancées. 
Les courbes en traits pleins des figures 3 et 4 correspondent aux meilleurs ajustements des valeurs de $K$ aux résultats expérimentaux pour différentes valeurs de la température.

La figure 5 montre que les rapports $K$ varient avec la température selon une loi d'Arrhénius ce qui semblerait prouver que les enthalpies libres des réactions de formation de $\mathrm{GaSb}$, InSb et $\mathrm{AlSb}$ évoluent d'une manière sensiblement identique avec la température. Cependant dans le domaine de température étudié, les valeurs des rapports $K$ ainsi obtenues sont très différentes de celles que l'on peut calculer à l'aide des données thermodynamiques de la littérature. Un tel désaccord a déjà été observé notamment dans la solution solide $\operatorname{InAs}_{1-x} \mathrm{Sb}_{x}[24$, 27]. La multiplication du $2^{\mathrm{e}}$ membre de (7) par un facteur correctif $\left(M_{\mathrm{In}} / M_{\mathrm{Ga}}\right)^{1 / 2}$ ou $\left(M_{\mathrm{Al}} / M_{\mathrm{Ga}}\right)^{1 / 2}$, pour tenir compte d'une vitesse de diffusion éventuellement différente des espèces gazeuses $\mathrm{Ga}$, In et Al, ne suffit pas pour compenser la différence (les $M_{i}$ sont les masses atomiques). Des réactions de surface ou une pyrolyse incomplète des organométalliques pourraient être responsables de ce désaccord.

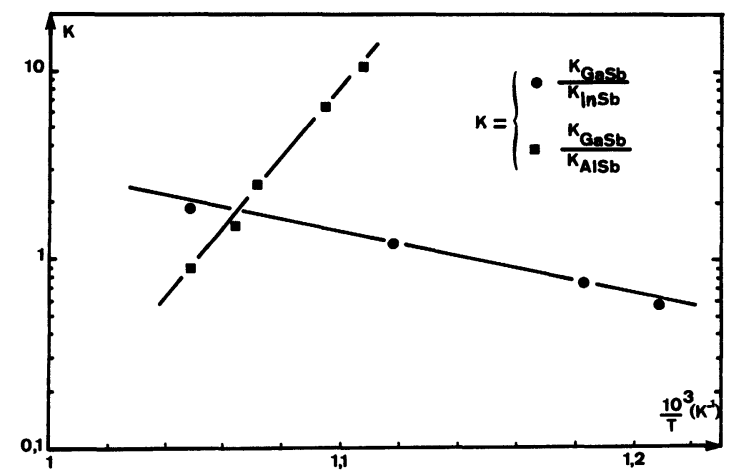

Fig. 5. - Evolution des rapports des constantes d'équilibres $K$ avec la température de croissance.

[Dependence of the equilibrium constants ratio $K$ on the growth temperature.]

$$
-\mathrm{GaAs}_{y} \mathrm{Sb}_{1-y} \text {. }
$$

Sur la solution solide ternaire $\mathrm{GaAs}_{y} \mathrm{Sb}_{1-y}$, seule a été examinée pour l'instant l'influence du rapport $R_{\mathrm{V} / \mathrm{III}}=P_{\mathrm{TMSb}}+P_{\mathrm{AsH}_{3}} / P_{\mathrm{TMG}}$ sur la relation liant $y$ à $y_{\mathrm{v}}$. Les résultats sont essentiellement décrits par la figure 6. Cette figure met en évidence le fait que, à $y_{\mathrm{v}}$ donné, la fraction atomique de GaAs dans le solide diminue lorsque le rapport éléments V/élément III diminue. On a reporté sur la même figure les valeurs expérimentales obtenues par Bedair et al. [11] dans un réacteur vertical sur substrats de GaAs avec un rapport $R_{\mathrm{V} / \text { III }}$ vraisemblablement égal à 3,43 et les valeurs calculées par Cherng [14] pour un mélange gazeux où TMAs remplace $\mathrm{AsH}_{3}$.

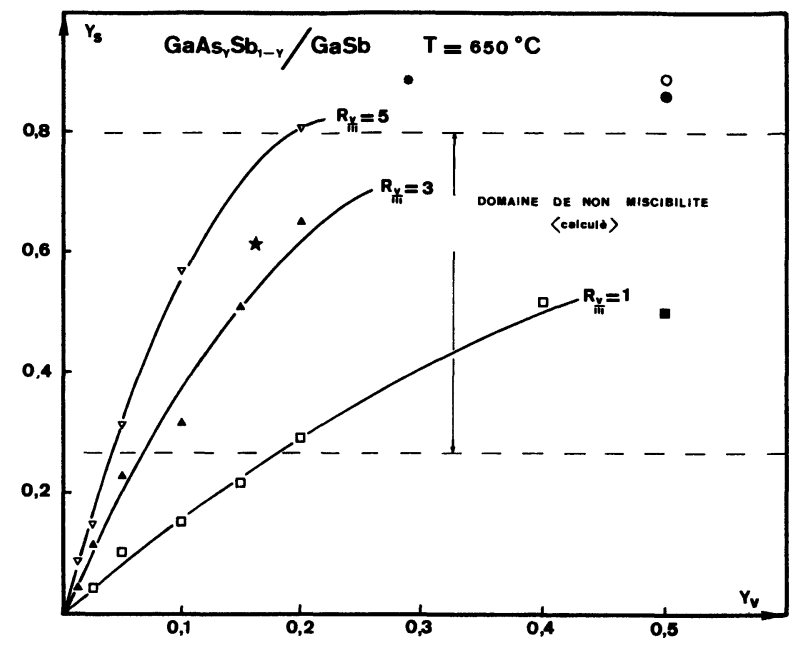

Fig. 6. - Fraction molaire $y_{\mathrm{s}}$ de GaAs dans le solide en fonction de $y_{\mathrm{v}}=P_{\mathrm{AsH}_{3}} / P_{\mathrm{AsH}_{3}}+P_{\mathrm{TMSb}}$, pour différentes valeurs de $R=P_{\mathrm{AsH}_{3}}+P_{\mathrm{TMSb}} / P_{\mathrm{TMG}}$.

valeurs calculées [14] : $\bigcirc R=5 ; \bullet R=3 ; \square R=1$

valeurs expérimentales [11]: $* R=3,43 ; \star R$ non donné; domaine de non-miscibilité calculé à $650^{\circ} \mathrm{C}$ d'après [4].

[GaAs molar fraction $y_{\mathrm{s}}$ in the solid as a function of $y_{\mathrm{v}}=P_{\mathrm{AsH}_{3}} / P_{\mathrm{AsH}_{3}}+P_{\mathrm{TMSb}}$, for different values of $R=$ $P_{\mathrm{AsH}_{3}}+P_{\mathrm{TMSb}} / P_{\mathrm{TMG}}$.

calculated values [14] : $O R=5 ; \bullet R=3 ; \square R=1$

experimental values [11] : $* R=3.43 ; \star R$ not given ; calculated miscibility gap at $650{ }^{\circ} \mathrm{C}$ from [4].]

Nos résultats sont qualitativement concordants en ce qui concerne la très rapide augmentation de $y$ quand $y_{\mathrm{v}}$ crôit et le rôle important du rapport $R_{\mathrm{V} / \text { III }}$ sur la composition du solide, à $y_{\mathrm{v}}$ donné ; le fait que le coefficient de ségrégation de l'arsenic $y_{\mathrm{s}} / y_{\mathrm{v}}$ soit nettement supérieur à 1 , contrairement à ceux de l'indium ou de l'aluminium dans les deux systèmes précédents, ne fait que traduire la plus grande stabilité de GaAs comparée à GaSb. Quantitativement, nos résultats sont difficilement comparables car Cherng est conduit à admettre une pyrolyse incomplète de TMAs et a travaillé avec des valeurs de $y_{\mathrm{v}}$ bien supérieures. L'influence de la température de croissance sur la composition du solide n'a pas encore été étudiée d'une manière systématique ; il semble qu'elle soit beaucoup plus faible que pour les deux autres solutions solides en accord avec Bedair et al. [11].

\section{Conclusion.}

Les solutions solides $\mathrm{Ga}_{1_{-}} \mathrm{Al}_{x} \mathrm{Sb}$ et $\mathrm{Ga}_{{ }_{-x}} \mathrm{In}_{x} \mathrm{Sb}$, avec $x \leqslant 0,5$, et $\mathrm{GaAs}_{y} \mathrm{Sb}_{y-1}$, avec $y \leqslant 0,81$, ont été déposées sur substrats de GaSb par EPVOM avec une morphologie satisfaisante tant que le désaccord de paramètre de maille reste inférieur $\frac{\Delta a}{a}=10^{-2}$.

Dans $\mathrm{Ga}_{1-x} \mathrm{Al}_{x} \mathrm{Sb}$ et $\mathrm{Ga}_{1-x} \mathrm{In}_{x} \mathrm{Sb}$, le rapport $x / x_{\mathrm{v}}$ est inférieur à 1 et il est sensible à la tempéra- 
ture de croissance dans le domaine étudié. De plus, à $x_{\mathrm{v}}$ donné, $x$ croît dans $\mathrm{Ga}_{1-x} \mathrm{Al}_{x} \mathrm{Sb}$ alors qu'il décroît dans $\mathrm{Ga}_{1-x} \mathrm{In}_{x} \mathrm{Sb}$ quand la température de croissance augmente.

Un modèle thermodynamique simple basé sur les hypothèses suivantes :

- pyrolyse complète des organométalliques et de l'arsine

- équilibre thermodynamique à l'interface solide-liquide

— vitesse de diffusion identique des espèces gazeuses

— solution solide régulière,

permet d'expliquer qualitativement cette évolution sans qu'un accord quantitatif puisse être obtenu en l'absence de données thermodynamiques sûres.
Dans $\mathrm{GaAs}_{y} \mathrm{Sb}_{1-y}$, le rapport $y / y_{\mathrm{v}}$ est supérieur en 1, en accord qualitatif avec la plus grande stabilité de GaAs comparé à $\mathrm{GaSb}$, et il semble être nettement dépendant du rapport $\mathrm{As}+\mathrm{Sb} / \mathrm{Ga}$ dans la phase vapeur. Il se confirme enfin une fois de plus que l'épitaxie en phase vapeur par pyrolyse d'organométalliques est capable de former la solution solide $\mathrm{GaAsSb}$ dans le domaine de non-miscibilité.

\section{Remerciements.}

Nous adressons nos remerciements à M. Gouskov pour le tirage des substrats de $\mathrm{GaSb}$, à $\mathrm{M}$. Fourcade, laboratoire de Chimie Minérale D, pour les mesures de diffraction de rayons $\mathrm{X}$ et à $\mathrm{MM}$. Rossi, Gril et Datas, laboratoire commun de Microscopie Electronique, pour les observations et mesures en microscopie électronique à balayage.

\section{Bibliographie}

[1] Gratton, M. F., Woolley, J. C., J. Electrochem. Soc. 127 (1980) 55.

[2] Nahori, R. E., Pollack, M. A., De Winter, J. C., Williams, K. M., J. Appl. Phys. 48 (1977) 1607.

[3] Fonstad, C. G., Quillec, M., Garone, S., J. Appl. Phys. 49 (1978) 5920.

[4] Pessetto, J. R., Stringfellow, G. B., J. Cryst. Growth 62 (1983) 1-6.

[5] De Cremoux, B., J. Physique Colloq. 43 (1982) C5 19.

[6] Marbeuf, A., Guillaume, J. C., J. Physique Colloq. 43 (1982) C5 47.

[7] Onabe, K., Jpn J. Appl. Phys. 21 (1982) 964.

[8] Stringfellow, G. B., J. Cryst. Growth 58 (1982) 194.

[9] Waho, T., Ogawa, S., Maruyama, S., Jpn J. Appl. Phys. 16 (1977) 1875.

[10] Chiu, T. H., Tsang, W. T., Ghu, S. N. G., Shan, J., Ditzenberger, J. A., Appl. Phys. Lett. 46 (1985) 408.

[11] Bedair, S. M., Timmons, M. L., Chiang, P. K., SimpSON, L., HAUSER, J. R., J. Electron. Mater. 12 (1983) 959.

[12] Stringfellow, G. B., Cherng, M. J., J. Cryst. Growth 64 (1983) 413.

[13] Cherng, M. J., Stringfellow, G. B., Appl. Phys. Lett. 44 (1984) 677.

[14] Cherng, M. J., Cohen, R. M., Stringfellow, G. B., J. Electron. Mater. 13 (1984) 799.
[15] Jen, H. R., Cherng, M. J., Stringfellow, G. B., Appl. Phys. Lett. 48 (1986) 1603.

[16] Cherng, M. J., Stringfellow, G. B., Appl. Phys. Lett. 48 (1986) 419.

[17] Dolginov, L. M., Drurhinina, L. V., Sov. J. Quantum Electron. 8 (1978) 416.

[18] De Winter, J. C., Pollack, M. A., J. Electron. Mater. 14 (1985) 729.

[19] Karouta, F., Thèse Montpellier 15 sept. (1986).

[20] Srivastava, A. K., De Winter, J. C., Caneau, C., Pollack, M. A., Zyskind, J. L., Appl. Phys. Lett. 48 (1986) 903.

[21] Quillec, M., Launois, H., Joncour, M. C., J. Vac. Sci. Technol. B 1 (1983) 238.

[22] GoryunOva, N. A., The chemistry of diamond like semi-conductors. (Chapman and Hall London) 1965.

[23] Bougnot, G., Foucaran, A., Marjan, M., Etienne, D., Bougnot, J., Delannoy, F., Roumanille, F., J. Cryst. Growth 77 (1986) 400.

[24] Fukui, T., Horikoshi, Y., Jpn J. Appl. Phys. 19 (1980) L53.

[25] Stringfellow, G. B., J. Cryst. Growth 27 (1974) 21.

[26] Stringfellow, G. B., J. Cryst. Growth 62 (1983) 225.

[27] Biefeld, R. M., J. Cryst. Growth 77 (1986) 392. 